\title{
The leukoaraiosis is more prevalent in the large artery atherosclerosis stroke subtype among Korean patients with ischemic stroke
}

\author{
Seung-Jae Lee ${ }^{1}$, Joong-Seok Kim*1, Kwang-Soo Lee ${ }^{1}$, Jae-Young An ${ }^{1}$, \\ Woojun Kim², Yeong-In Kim ${ }^{1}$, Bum-Soo Kim ${ }^{3}$ and So-Lyung Jung ${ }^{3}$
}

Address: ${ }^{1}$ Department of Neurology, The Catholic University of Korea, Seoul, South Korea, ${ }^{2}$ Department of Neurology, National Cancer Center, Koyang-city, Kyunggi-province, South Korea and ${ }^{3}$ Department of Radiology, The Catholic University of Korea, Seoul, South Korea

Email: Seung-Jae Lee - neurosj@catholic.ac.kr; Joong-Seok Kim* - neuronet@catholic.ac.kr; Kwang-Soo Lee - ks1007@catholic.ac.kr; JaeYoung An - musclenerve@catholic.ac.kr; Woojun Kim - wjkim@catholic.ac.kr; Yeong-In Kim - nuyikim@catholic.ac.kr; BumSoo Kim - bumrad@catholic.ac.kr; So-Lyung Jung - sljung1@catholic.ac.kr

* Corresponding author

Published: 7 August 2008

BMC Neurology 2008, 8:31 doi:|0.||186/|47|-2377-8-3|
Received: 12 March 2008

Accepted: 7 August 2008

This article is available from: http://www.biomedcentral.com/I47I-2377/8/3 I

(C) 2008 Lee et al; licensee BioMed Central Ltd.

This is an Open Access article distributed under the terms of the Creative Commons Attribution License (http://creativecommons.org/licenses/by/2.0), which permits unrestricted use, distribution, and reproduction in any medium, provided the original work is properly cited.

\begin{abstract}
Background: Several studies have suggested that the specific stroke subtype may influence the presence of leukoaraiosis in patients with ischemic stroke. We investigated the association between stroke subtype and leukoaraiosis in Korean patients with ischemic stroke by MRI.
\end{abstract}

Methods: There were 594 patients included in this study that were classified as large artery disease, lacune and cardioembolic stroke. For large-artery disease, the analysis focused on the intracranial or extracranial location of the stenosis, and the multiplicity of the stenotic lesions. Leukoaraiosis grading was performed according to the Atherosclerosis Risk in Communities Study.

Results: There was a significant association between leukoaraiosis and the stroke subtypes; the large-artery-disease group had a higher prevalence of leukoaraiosis than did the other groups (55.4\% in the large-artery-disease group, $30.3 \%$ in the lacunar group and $14.3 \%$ in the cardioembolic group, $P=0.016$ by chi-square test). On the multivariate linear regression analysis, age, the presence of hypertension, previous stroke and stroke subtype were independently associated with the presence of leukoaraiosis. In the sub analysis of the large-artery-disease group, the leukoaraiosis had a tendency to be more prevalent in the mixed and intracranial stenosis group than did the extracranial stenosis group ( $45.5 \%$ in the mixed group, $40.3 \%$ in the intracranial group and $26.9 \%$ in the extracranial group, $\mathrm{P}=0.08$ by chi-square test).

Conclusion: The association of leukoaraiosis with large-artery disease in this study might be due to the relatively high prevalence of intracranial occlusive lesions in Korean stroke patients compared to other ethnic groups.

\section{Background}

The term leukoaraiosis (LA) refers to lesions of altered signal intensity on computed tomography (CT) and mag- netic resonance imaging (MRI) in the periventricular and subcortical white matter. LA is found during the normal aging process, and in the patients with cerebrovascular 
disease. It also constitutes the core pathology of Binswanger's disease, a type of vascular dementia. The association of LA with lacunar infarcts rather than territorial infarcts is well documented [1-7]. However, most prior studies have been based on CT findings, not MRI, and reported from western countries.

In the present study, we analyzed the association between stroke subtype and LA in Korean stroke patients using MRI.

\section{Methods}

\section{Patients}

We initially included 963 consecutive acute ischemic stroke patients admitted to the neurology department from July 2003 to June 2007. All patients underwent detailed clinical evaluation including laboratory tests, chest radiography, transcranial Doppler study, electrocardiography and 24 hour Holter monitoring. In addition, transthoracic echocardiography and brain magnetic resonance imaging (MRI), contrast-enhanced MR angiography (MRA) and/or cerebral angiography were obtained. All results from the evaluations were analyzed according to the diagnostic criteria for stroke mechanisms and etiology based on the TOAST subtype classification system [8]. Among the initial patients, 369 were categorized into stroke of undetermined etiology (mean age \pm SD, $68.1 \pm$ 10.2; age range, 31-87 years) and were excluded from the study: of those patients, 245 patients were classified as stroke of two or more potential etiology (164 with lacune plus large-artery disease, 54 with large-artery disease plus cardioemobolism and 27 with lacune plus cardioembolism), 21, 76 and 27 patients were classified as groups of negative evaluation, incomplete evaluation and other determined etiology, respectively.

Finally, 594 cases with large artery disease (297 patients), lacune (193 patients) and cardioembolic stroke (104 patients) were enrolled in this study. The ethics committee at our institution approved the study protocol, and all subjects provided written informed consent.

\section{Risk factor evaluation}

The clinical information included age, gender, history of hypertension (defined by the use of an antihypertensive agent before admission or a systolic pressure $>140 \mathrm{mmHg}$ or diastolic pressure $>90 \mathrm{mmHg}$ demonstrated on repeated examinations at least one month after presentation with a stroke), diabetes mellitus (defined as a fasting blood glucose level $>126 \mathrm{mg} / \mathrm{dl}$ or a history of being treated for diabetes mellitus) and hyperlipidemia (defined as a total cholesterol level $>200 \mathrm{mg} / \mathrm{dl}$ or a lowdensity lipoprotein cholesterol $>130 \mathrm{mg} / \mathrm{dl}$ at the time of presentation or a history of treatment). In addition, regular cigarette smoking, a previous history of ischemic stroke and heart disease (defined as a known history or clinical demonstration of any heart disease, including myocardial infarction, angina pectoris, congestive heart failure, or arrhythmia) were noted

\section{MR imaging and $L A$ grading}

All patients enrolled underwent conventional MRI on a 1.5-T system (Signa 1.5-T TwinSpeed, General Electric Medical Systems and Archieva 1.5-T, Philips Electronics) within 7 days of the stroke onset. The conventional MRI consisted of transverse T2/T1-weighted, fluid-attenuated inversion recovery (FLAIR) sequences and sagittal T1 with 5-mm-thick slices. Diffusion-weighted imaging was obtained in the transeverse plane using a single-shot echoplanar, spin-echo pulse sequence. A three-dimensional time of flight MRA of the intracranial arteries and contrast-enhanced MRA of the head and neck were also performed on the same system using a neurovascular coil.

LA was defined as a periventricular white matter lesion with hyperintensity on T2- weighted and FLAIR images and without prominent hypointensity on T1-weighted images. The LA grading was according to the Atherosclerosis Risk in Communities (ARIC) study $[9,10]$. Three trained neurologists and two neuroradiologists, blinded to patient data and stroke subtype, graded the LA by consensus. When evaluating the WMH, new (high signal on diffusion-weighted image) and old (definitely low signal on T1-weighted image) infarcts were excluded. If one or both sides of the brain were focally abnormal, estimates were based on the uninvolved side with the principle of symmetry assumed.

\section{Patterns of stenotic lesions in large artery disease}

The evaluation of the arterial stenosis was performed by the investigators and $>50 \%$ of signal loss on the MRA was considered to be a "significant stenosis" for the classification of stroke subtype and the categorization of stenosis pattern. The locations of significant stenosis were categorized as located in the intracranial or extracranial arteries. For the internal carotid artery, an intracranial location was defined when the stenotic lesion was distal to the ophthalmic artery. For the vertebral artery, the differentiation was made at the point where the artery pierced the dura at the level of the foramen magnum. The intracranial extent of the stenosis included up to the M2 of the middle cerebral artery (insular segment which terminates at the circular sulcus of the insula before the operculum) and the A2 segment of the anterior cerebral artery (ascending segment with inferior forward convexity) in the anterior circulation, and the P2 segment of the posterior cerebral artery (ambient segment which extends from the junction between the posterior communicating artery and the posterior cerebral artery to the posterior aspect of midbrain). The area of the stenotic lesion was divided into intracra- 
nial or extracranial and anterior or posterior circulation. The stenoses were described as single or multiple according to the number of the areas involved with the arterial stenosis.

According to the distribution and pattern of the stenotic lesions, the patients with large-artery disease were categorized as intracranial, extracranial and a mixed (intracranial plus extracranial) group, and as multiple- or singlestenosis groups.

\section{Statistical analysis}

We classified persons with leukoaraiosis of grade 3 or higher as having "LA" and of grade 2 or lower as having "little or no LA" [10]. The presence or absence of LA was compared between the patients with risk factors and the patients without, the stroke subtypes, the multiple stenosis and single stenosis groups, and among intracranial, mixed and extracranial stenosis groups by chi-square test or independent t-test. In addition, we compared the severity of LA between the groups of different stroke subtypes by one-way ANOVA test and LSD multiple comparison test. Multiple linear regression analysis was used to determine the factors considered independently associated with leukoaraiosis. Values of $\mathrm{P}<0.05$ were considered statistically significant.

\section{Results}

Of the 594 patients, 342 (57.6\%) were men and 252 $(42.4 \%)$ were women (mean age \pm SD, $66.8 \pm 12.1$; age range, 27-97 years). In study population, distinct white matter changes were present in 307 patients (51.7\%). The association of the presence of LA to age was statistically significant; the LA group had higher age at onset of stroke than the "little or no LA" group. The LA was more frequently observed in female gender, the patients with hypertension and a history of previous ischemic stroke than in the patients without this history $(\mathrm{P}<0.05)$. There was no significant association between the LA and diabetes mellitus or hyperlipidemia; a negative correlation was found with the smoking status and the presence of heart disease (table 1).

Among the 307 patients with LA, 170 patients (55.4\%) were in the large-artery-disease group, 93 patients $(30.3 \%)$ in the lacunar group and 44 patients $(14.3 \%)$ in the cardioembolic group $(\mathrm{P}=0.016$ by chi-square test, table 1). In addition, there was overall a significant association between the LA grade and the stroke subtype: the large-artery-disease group had more severe LA disease than did the other groups. Although the lacunar group tended to have a higher LA grade than did the cardioembolic group, there was no significant difference in the LA grade in comparisons between the two groups (table 2 ). On the multivariate linear regression analysis, using the variables (age, gender, the presence of hypertension, diabetes mellitus, hyperlipidemia, smoking, previous stroke, and stroke subtype), age, the presence of hypertension, previous stroke and stroke subtype were independently associated with the presence of LA (Table 3 ).

In the large-artery-disease group, there was a borderline association between the LA and the stenotic areas; the LA was most prevalent in the mixed stenotic group, next was the intracranial stenosis group and the extracranial stenosis group was the least affected. In addition, a more LA was

Table I: Characteristics of stroke patients by leukoaraiosis.

\begin{tabular}{|c|c|c|c|c|}
\hline \multirow[b]{2}{*}{ Characteristics } & \multicolumn{2}{|c|}{ Leukoaraiosis } & \multirow[b]{2}{*}{$P$ value } & \\
\hline & Present $(n=307)$ & Absent $(n=287)$ & & \\
\hline Age $(y r)$ & $72.1 \pm 9.8$ & $61.1 \pm 11.9$ & $<0.001$ & \\
\hline Gender & & & 0.001 & \\
\hline Male $(n=342)$ & $157(45.9)$ & $185(54.1)$ & & \\
\hline Female $(n=252)$ & $150(59.5)$ & $102(40.5)$ & & \\
\hline Hypertension $(n=383)$ & $229(59.8)$ & $154(40.2)$ & $<0.001$ & \\
\hline Diabetes mellitus $(n=236)$ & $133(56.4)$ & $103(43.6)$ & 0.390 & \\
\hline Hyperlipidemia $(n=193)$ & $95(49.2)$ & $98(50.8)$ & 0.228 & \\
\hline Heart disease $(n=105)$ & 44 (41.9) & $61(58.1)$ & 0.018 & \\
\hline Atrial fibrillation $(n=78)$ & $34(43.6)$ & $44(56.4)$ & 0.125 & \\
\hline Regular cigarette smoking $(n=153)$ & $57(37.3)$ & $96(62.7)$ & $<0.001$ & \\
\hline Previous ischemic stroke $(n=87)$ & $58(66.7)$ & $29(33.3)$ & 0.002 & \\
\hline Stroke subtype & & & 0.016 & \\
\hline Large artery disease $(\mathrm{n}=297)$ & $170(57.2)$ & $127(42.8)$ & $0.03 I^{a}$ & $0.006^{b}$ \\
\hline Lacunar $(n=193)$ & $93(48.2)$ & $100(51.8)$ & $0.198^{b}$ & \\
\hline Cardioembolic $(n=104)$ & $44(42.3)$ & $60(57.7)$ & & \\
\hline
\end{tabular}

Values represent mean \pm SD and number of patients with percentages in parenthesis

The two groups were compared by two-sample $t$-test for continuous variables and chi-square test for nominal variables.

a Comparison is with lacunar group, based on the Chi-square test.

b Comparison is with cardioembolic group 
Table 2: Leukoaraiosis grade by stroke subtype.

\begin{tabular}{cccc}
\hline & $\begin{array}{c}\text { Large artery disease } \\
(\mathrm{n}=297)\end{array}$ & $\begin{array}{c}\text { Stroke subtype } \\
\text { Lacunar } \\
(\mathrm{n}=193)\end{array}$ & $\begin{array}{c}\text { Cardioembolic } \\
(\mathrm{n}=104)\end{array}$ \\
\hline $\begin{array}{c}\text { LA grade } \\
(\text { mean } \pm \text { SD }) \\
\text { LSD, } P \text { value }\end{array}$ & $3.37 \pm 1.98$ & $2.86 \pm 1.95$ & $2.54 \pm 1.71$ \\
\hline
\end{tabular}

$P$ value refers to the overall association between leukoaraiosis grade and stroke subtype, computed from one-way ANOVA test. a Comparison is with the lacunar group, based on LSD multiple comparison tests.

b Comparison is with the cardioembolic group

observed in patients with multiple arterial stenoses than in patients with single arterial stenosis (table 4).

\section{Discussion}

Although the pathophysiology of LA remains speculative, there is evidence to suggest that LA may be linked to cerebral ischemia [11-24]. Selective injury to the cerebral white matter has been noted in a limited number of human conditions characterized by hypoxia/ischemia of the brain such as carbon monoxide poisoning and therapeutic occlusion of the internal carotid artery [11-14]. It has been assumed that the ischemic insult, responsible for LA, results from the vulnerable nature of the long penetrating end-arteries that feed the deep white matter $[15,16]$. LA has been associated with increasing age, arterial hypertension and other cerebrovascular risk factors [17-22]. In addition, white matter lesions similar to LA can be induced in rats or gerbils by ligating the bilateral common carotid arteries [23,24].

Although the previous studies of risk factors for LA have shown different results, advanced age and hypertension have been consistently reported to be highly associated with LA [1]. One recent study indicated that diabetes mellitus seems to be a risk factor for progression rather than new LA development [7]. In our study, similar to previous studies in other ethnic groups, LA was associated with age, hypertension and previous history of ischemic stroke.

Table 3: Multiple linear regression analysis for the relationship between leukoaraiosis and potential confounding variables.

\begin{tabular}{lcc}
\hline & $t$ & $p$ \\
\hline Age & 11.130 & $<0.001$ \\
Gender & 1.056 & 0.292 \\
Hypertension & 4.132 & $<0.001$ \\
Diabetes mellitus & 0.611 & 0.541 \\
Hyperlipidemia & -0.117 & 0.907 \\
Smoking & 0.550 & 0.583 \\
Previous stroke & 2.440 & 0.015 \\
Stroke subtype & 2.449 & 0.013
\end{tabular}

Data are $t$ ( $P$ value) of the correlation.

$\mathrm{aR}^{2}=0.503$
However, it had no relation with hyperlipidemia and diabetes mellitus. In addition, there was a negative correlation between regular cigarette smoking and LA, as previously reported in one study [21]. However, the relationship between smoking and brain disorder is controversial, and the negative association with LA shown in this study should be discussed in further future study. These differences between the results of previous researches may be due to study variations in patients and/or the definitions of LA used by different investigators.

The LA was more frequently observed in the large-arterydisease group than the other subtypes. In addition, the large-artery-disease group had more severe LA than did the other group. The cardioembolic group had the lowest prevalence of LA although there was no significant difference when compared to the lacunar group. This finding suggests that the hypoperfusion that results from largeartery occlusion might be more important to the progression and aggravation of LA. This is supported by the fact that the periventricular white matter, vulnerable to LA, was the distal irrigation field or border zone; these areas are prone to ischemia under conditions of moderate blood flow deficits.

However, the results of our study are in general not consistent with prior reports. In most previously reported studies, LA was strongly associated with lacunar strokes rather than non-lacunar, territorial strokes [1-7]. In addition, an inverse correlation between high-grade (> 50\%) stenoses of the extracranial arteries and white matter changes has been reported $[1,21,25-27]$. These observations have been explained by the hypothesis that reduced blood perfusion, in the patients with high-grade stenoses of the extracranial carotid arteries, might alleviate damage to intracerebral arteries by decreasing tensile stress on the arterial walls $[21,28]$. However, these studies did not divide the territorial infarcts into large artery disease or cardioembolic strokes. Moreover, most prior studies were from western countries, and the results were based on CT findings not MRI, which is better for assessing white matter lesions. 
Table 4: Association between the pattern of stenosis and leukoaraiosis in large-artery-disease group.

\begin{tabular}{|c|c|c|c|c|}
\hline & \multicolumn{2}{|c|}{ Leukoaraiosis } & \multirow[b]{2}{*}{$P$ value } & \\
\hline & Present $(n=170)$ & Absent $(n=127)$ & & \\
\hline Distribution of stenosis & & & 0.080 & \\
\hline Mixed $(n=101)$ & $46(45.5)$ & $55(54.5)$ & $0.245^{a}$ & $0.023^{b}$ \\
\hline Intracranial $(n=144)$ & $58(40.3)$ & $86(59.7)$ & $0.070^{b}$ & \\
\hline Extracranial $(n=52)$ & $14(26.9)$ & $38(73.1)$ & & \\
\hline Number of stenotic lesions & & & 0.019 & \\
\hline Multiple $(n=155)$ & $71(45.8)$ & $84(54.2)$ & & \\
\hline Single $(n=142)$ & $47(33.1)$ & $95(66.9)$ & & \\
\hline
\end{tabular}

Values represent number of patients with percentages in parenthesis The two groups were compared by chi-square test.

a Comparison is with intracranial group

b Comparison is with extracranial group

In the present study, most of the patients in the largeartery-disease group had intracranial stenotic lesions $(82.5 \%)$; extracranial lesions were uncommon. The LA tended to be more frequent in patients with intracranial stenoses than in the patients with extracranial stenoses alone. The intracranial location for cerebrovascular atherosclerosis is characteristic of strokes in the Asian population [29-31]. Therefore, the more prevalent LA in patients with large-artery-disease compared to the other subtypes in this study might be explained by the high prevalence of intracranial stenoses in Korean stroke patients.

Unlike extracranial stenoses, more prevalent in Caucasians, intracranial stenoses may have a different pathogenesis contributing to the development and progression of LA. First, atherosclerotic stenoses of intracranial arteries can directly occlude the orifice of numerous small perforators penetrating into the deep brain parenchyma. The occlusion of the small perforators promotes extensive hypoperfusion of the periventricular region that is vulnerable to ischemia. Second, the periventricular border zone, under ischemic conditions induced by the stenoses of intracranial arteries, might have less opportunity to be compensated by blood flow via major collateral channels such as the anterior or posterior communicating artery, which can be recruited without difficulty in stroke patients with stenosis of extracranial vessels. Third, compared with the emboli from the stenotic lesions of extracranial arteries, those from an intracranial location might not easily be cleared away by travel along with the blood flow, probably because of the close proximity to the perfused brain tissues.

The limitations of this study include the following. One methodological problem is that our results are based on a cross-sectional sample. The longitudinal effect of largeartery atherosclerosis on periventricular white matter changes could not be accurately assessed in this study. In addition, a selection bias might have been present because $369(38.3 \%)$ of the 963 patients were excluded due to the diagnosis of a stroke of undetermined etiology or other determined etiology; we used very strict criteria for the classification of subjects into specific stoke subtypes. For example, suspected large artery disease with < $50 \%$ signal loss of the proximal artery on MRA, or supratentorial subcortical infarcts of $<1.5 \mathrm{~cm}$ with $>50 \%$ stenosis of the parent artery were classified as strokes of undetermined origin. The application of this criterion would increase the specificity and lessen the likelihood of misclassification of patients in the other categories [8]. Moreover, the quantitative analysis of LA by a 0-to- 9 grading system might not reflect an accurate estimation of the LA severity. Additional grading systems including volumetric methods for LA are needed.

\section{Conclusion}

The results of this study showed that LA is significantly associated with large-artery-disease rather than other stroke subtypes including small vessel disease in Korean stroke patients. The differences between our study and previous reports might be due to the high prevalence of intracranial occlusive lesions in patients with cerebrovascular atherosclerosis in the Asian population. Different from the high-grade stenoses of extracranial arteries, the stenoses of intracranial arteries might induce extensive white matter changes by interrupting blood flow to the periventricular border zone vulnerable to ischemia.

\section{Competing interests}

The authors declare that they have no competing interests.

\section{Authors' contributions}

SJL and JSK conceived and coordinated the study, analysed the data and drafted the initial manuscript. All authors were involved in initial literature search and col- 
lection of data. Review of initial manuscript for major intellectual content was done by SJL and JSK. All authors read and approved the final manuscript.

\section{Acknowledgements}

This study was supported by a grant of the Korea Health 2I R\&D Project, Ministry of Health and Welfare, Republic of Korea (project no. A060093).

\section{References}

I. Leys D, Englund E, Del Ser T, Inzitari D, Fazekas F, Bornstein N, Erkinjuntti T, Bowler JV, Pantoni L, Parnetti L, De Reuck J, Ferro J, Bogousslavsky J: White matter changes in stroke patients. Relationship with stroke subtype and outcome. Eur Neurol 1999, 42:67-75.

2. Hijdra A, Verbeeten B Jr, Verhulst JA: Relation of leukoaraiosis to lesion type in stroke patients. Stroke 1990, $21: 890-894$.

3. Leys D, Pruvo JP, Scheltens P, Rondepierre P, Godefroy O, Leclerc X, de Reuck J: Leukoaraiosis: Relationship with the types of focal lesions occurring in acute cerebrovascular disorders. Cerebrovasc Dis 1992, 2:169-176.

4. Hijdra A, Verbeeten B Jr: Leukoaraiosis and ventricular enlargement in patients with ischemic stroke. Stroke I991, 22:447-450

5. Jayakumar PN, Taly AB, Shanmugan V, Nagarja D, Arya BYT: Mult infarct dementia: A computed tomographic study. Acta Neurol Scand 1989, 73:292-295.

6. Boon A, Lodder J, Heuts-van Raak L, Kessels F: Silent brain infarcts in 755 consecutive patients with a first-ever supratentorial ischemic stroke: Relationship with index-stroke subtype, vascular risk factors, and mortality. Stroke 1994, 25:2384-2390.

7. Gouw AA, Flier WM van der, Fazekas F, van Straaten EC, Pantoni $L$, Poggesi A, Inzitari D, Erkinjuntti T, Wahlund LO, Waldemar G, Schmidt R, Scheltens P, Barkhof F, LADIS Study Group: Progression of white matter hyperintensities and incidence of new lacunes over a 3-year period: the Leukoaraiosis and Disability study. Stroke 2008, 39:1414-20.

8. Adams HP Jr, Bendixen BH, Kappelle LJ, Biller J, Love BB, Gordon DL, Marsh EE 3rd: Classification of subtype of acute ischemic stroke. Definitions for use in a multicenter clinical trial. TOAST. Trial of Org 10172 in Acute Stroke Treatment. Stroke 1993, 24:35-4I.

9. Liao D, Cooper L, Cai J, Toole JF, Bryan NR, Hutchinson RG, Tyroler HA: Presence and severity of cerebral white matter lesions and hypertension, its treatment, and its control. The ARIC Study. Atherosclerosis Risk in Communities Study. Stroke 1996, 27:2262-70

10. Wong TY, Klein R, Sharrett AR, Couper DJ, Klein BE, Liao DP, Hubbard LD, Mosley TH, ARIC investigators: Atherosclerosis Risk in Communities Study. Cerebral white matter lesions, retinopathy, and incident clinical stroke. JAMA 2002, 288:67-74.

II. Brucher JM: Leukoencephalopathy in anoxic-ischemic processes. In Handbook of Clinical Neurology. Demyelinating Diseases Volume 3. Issue 47 Edited by: Koetsier JC. Amsterdam, Netherlands: Elsevier Science Publishers BV; 1985:525-549.

12. Ginsberg MD, Hedley-Whyte ET, Richardson EP: Hypoxicischemic leukoencephalopathy in man. Arch Neurol 1976, 33:5-14.

13. Schwedenberg TH: Leukoencephalopathy following carbon monoxide asphyxia. I Neuropathol Exp Neurol 1959, 1 8:597-608.

14. Awad IA, Masaryk T, Magdinec M: Pathogenesis of subcortical hyperintense lesions on MRI of the brain. Observations in patients undergoing controlled ICA occlusion. Stroke 1993, 24:1339-1346.

15. O'Sullivan M, Lythgoe DJ, Pereira AC, Summers PE, Jarosz JM, Williams SC, Markus HS: Patterns of cerebral blood flow reduction in patients with ischemic leukoaraiosis. Neurology 2002, 59:32I-326

16. Miyazawa N, Satoh T, Hashizume K, Fukamachi A: Xenon contrast CT-CBF measurements in high-intensity foci on T2weighted MR images in centrum semiovale of asymptomatic individuals. Stroke 1997, 28:984-987.

17. Jorgensen HS, Nakayama H, Raaschou HO, Olsen TS: Leukoaraiosis in stroke patients: the Copenhagen Stroke Study. Stroke 1995, 26:588-592.
I8. van Zagten M, Boiten J, Kessels F, Lodder J: Significant progression of white matter lesions and small deep (lacunar) infarcts in patients with stroke. Arch Neurol 1996, 53:650-655.

19. Raiha I, Tarvonen S, Kurki T, Rajala T, Sourander L: Relationship between vascular factors and white matter low attenuation of the brain. Acta Neurol Scand 1993, 87:286-289.

20. Ylikoski A, Erkinjuntti T, Raininko R, Sarna S, Sulkava R, Tilvis R White matter hyper in tensities on MRI in the neurologically nond is eased elderly. Analysis of cohorts of consecutive subjects aged 55 to 85 years living at home. Stroke 1995, 26:1 I7I-II77.

21. Wiszniewska M, Devuyst G, Bogousslavsky J, Ghika J, van Melle G: What is the significance of leukoaraiosis in patients with acute ischemic stroke? Arch Neurol 2000, 57:967-973.

22. Coskun O, Yildiz H, Emre U, Akin Ü, Üçler S, Ergun U, Tunç T, Inan EL: Leukoaraiosis in stroke patients. Int J Neurosci 2003, I I 3:915-922.

23. Wakita $\mathrm{H}$, Tomimoto $\mathrm{H}$, Akiguchi I, Kimura J: Glial activation and white matter changes in the rat brain induced by chronic cerebral hypoperfusion: an immunohistochemical study. Acta Neuropathol 1994, 87:484-492.

24. Kurumatani T, Kudo T, Ikura $Y$, Takeda M: White matter changes in the gerbil brain under chronic cerebral hypoperfusion. Stroke 1998, 29:1058-1062

25. Bogousslavsky J, Regli F, Uske A: Leukoaraiosis in patients with ischemic stroke. Stroke 1987, 18:896-899.

26. Streifler JY, Eliasziw M, Benavente OR, Hachinski VC, Fox AJ, Barnett $\mathrm{HJ}$ : Lack of relationship between leukoaraiosis and carotid artery disease. The North American Symptomatic Carotid Endarterectomy Trial. Arch Neurol 1995, 52:21-24.

27. Fazekas F, Niederkorn K, Schmidt R, Offenbacher H, Horner S, Bertha $\mathrm{G}$, Lechner $\mathrm{H}$ : White matter signal abnormalities in normal individuals: correlation with carotid ultrasonography, cerebral blood flow measurements, and cerebrovascular risk factors. Stroke 1988, 19:1285-1288.

28. Masawa N, Yoshida Y, Yamada T, Joshita T, Sato S, Mihara B: Morphometry of structural preservation of tunica media in aged and hypertensive human intracerebral arteries. Stroke 1994, 25: $122-127$

29. Sacco RL, Kargman DE, Gu Q, Zamanillo MC: Race-ethnicity and determinants of intracranial atherosclerotic cerebral infarction: The Northern Manhattan Stroke Study. Stroke 1995, 26: $14-20$.

30. Feldmann E, Daneault N, Kwan E, Ho KJ, Pessin MS, Langenberg P, Caplan LR: Chinese-white differences in the distribution of occlusive cerebrovascular disease. Neurology 1990, 40:154I-I545.

31. Suh DC, Lee SH, Kim KR, Park ST, Lim SM, Kim SJ, Choi CG, Lee HK: Pattern of atherosclerotic carotid stenosis in Korean patients with stroke: Different involvement of intracranial versus extracranial vessels. Am J Neuroradiol 2003, 24:239-244.

\section{Pre-publication history}

The pre-publication history for this paper can be accessed here:

http://www.biomedcentral.com/1471-2377/8/31/prepub 